\title{
INDEX TO VOLUME 62
}

\section{Subjects:}

\section{INDEX OF ABSTRACTS}

\author{
Algebra and Theory of Numbers, 1, 13, 24, 37, 148, 236, 348, 375, 401, 546. \\ Analysis, 5, 15, 28, 39, 154, 243, 352, 380, 405, 560. \\ Applied Mathematics, 8, 19, 30, 44, 166, 253, 359, 391, 409, 585. \\ Geometry, 10, 21, 45, 170, 256, 360, 395, 410, 594. \\ Logic and Foundations, 175, 260, 362, 395, 411, 598. \\ Statistics and Probability, 46, 176, 263, 363, 397, 413, 602. \\ Topology, 10, 22, 33, 47, 178, 264, 364, 397, 414, 605.
}

Authors:

Abhyankar, S. S., 375; Ablow, C. M., 585; Agmon, S., 154; Akers, S. B., Jr., 395; Akutowicz, E. J., 405; Albert, A. A., 348; Anderson, D. R., 560; Anderson, F. W., 364, 364; Anderson, L. W., 33, 178, 605; Anderson, R. D., 178, 178, 264, 397, 605, 605; Andrushkiw, J., 243; Ankeny, N. C., 546; Arens, R., 561; Armentrout, S., 265; Arms, R. J., 253; Arnold, H., 265; Aronson, D. G., 561; Aronszajn, N., 154; Arsove, M. G., 15, 380, 561; Auslander, L., 256; Austin, D. G., 263.

Bachman, G., 375; Bade, W. G., 15; Baer, R. M., 236, 348; Bagley, R. W., 179; Baker, G. A., Jr., 175, 175, 175, 602; Baker, G. A., Sr., 602; Banaschewski, B., 22, 265; Band, W., 586; Bareiss, E. H., 391, 586; Barrar, R. B., 243; Barrett, J. H., 155, 561; Barrett, L. K., 179; Barsotti, I., 410; Bass, R. W., 155, 155, 156, 166, 352, 352, 359; Baum, J. D., 398; Baxter, G. E., 46, 176, 363, 602; Baxter, W. E., 1, 376; Bear, H. S., 562; Beck, A., 243; Beckenbach, E. F., 156; Becker, H. W., 546, 586; Bendat, J. S., 586; Berberian, S. K., 348; Bergman, S., 39, 166, 381, 562, 562; Berman, G., 547, 547; Bharucha-Reid, A. T., 414, 602; Bing, R. H., 47, 47, 179, 365, 605; Birkhoff, G., 156; Blackett, D. W., 236; Blackman, J., 381; Blank, A. A., 253, 263; Blair, R. L., 364, 364; Block, R., 349; Blumenthal, L. M., 170, 594; Bohun-Chudnyniv, V., 548; Bonsall, F. F., 5; Bonne, W. W., 148; Boyer, D. L., 13; Bradshaw, C. L., 6; Brahana, T. R., 33; Brauer, A. T., 24; Brauer, G. U., 353, 563; Bremermann, H. J., 243; Brenner, J. L., 149, 149, 547, 586; Briggs, C. F., 365; Brock, R. L., 587; Browder, F. E., 5, 381; Brown, A., 156; Brown, E. H., Jr., 365; Brownell, F. H., 405, 587; Bruck, R. H., 548; Brunk, H. D., 40, 156, 157; Büchi, J. R., 237; Bryant, S. J., 349; Buck, R. C., 244, 244; Buehler, R. J., 46; Butler, J. W., 401.

Cairns, S. S., 170, 177; Calabi, E., 360; Cameron, R. H., 157; Campbell, H. E., 1; Campbell, S. G., 30; Capel, C. E., 33, 33; Carlitz, L., 24, 25, 25; Carr, J. W., 588; Carrier, G. F., 392; Cassity, C. R., 589; Cesari, L., 244, 382; Chacon, R. V. S., 263; Chamberlin, R. E., 606; Chambré, P. L., 588; Chang, C. C., 411, 598, 599; Chen, K. T., 37, 382, 382; Chihara, T. S., 563; Choquet, G., 178; Chung, K. L., 177; Civin, P., 157, 245; Clement, P. A., 586; Clifford, A. H., 25; Coburn, N., 392; Coddington, E. A., 405, 406, 406; Cohen, E., 2, 25, 548, 548; Cohen, H., 34, 606; Cohn, H., 237, 549; Colquitt, L. A., 167; Conrad, P. F., 26; Conroy, M. F., 253; Conte, S. D., 32; Copeland, A. H., Jr., 149, 180; Corson, H. H., 25; Court, N. A., 171; Cowell, W. R., 594; Cox, J. G., 6; Craig, W., 411; Crowe, D. W., 549; Crowell, R. H., 149, 265; Curtis, M. L., 180, 180; Curtis, P. C., Jr., 563; Cuthill, E., 392.

Davis, A. C., 401, 411; Davis, C., 245; Davis, R. B., 5; Davis, R. L., 549; Davis, R. M., 392; Davison, W. F., 180; DeCicco, J., 349, 360; Day, M. M., 45, 549, 550, 563; Deskins, W. E., 150; Diamond, A. H., 167; Diaz, J. B., 253; De Prima, R. C., 
392; Diliberto, S. P., 563, 564; Dilworth, R. P., 550; Divinsky, N. J., 38, 550, 659; Donsker, M. D., 176, 363; Douglas, J., Jr., 168, 564, 588, 588; Downing, A. C., Jr., 564; Doyle, T. C., 589; Dvoretzky, A., 602; Dyer, E., 10, 181, 266, 266, 398.

Eberlein, W. F., 382; Edmondson, D. E., 349; Edrei, A., 158, 245, 383; Eells, J., Jr., 257, 257; Eisenstadt, B. J., 564, 565; Elgot, C. C., 44; Elkins, T. A., 353; Ellis, D. O., 550; Epstein, I. J., 392; Epstein, M. P., 237; Ergen, W. K., 29, 29; Eringen, A. C., 45; Evans, T., 2; Ewing, G. M., 40, 157.

Fadell, E. R., 365; Faith, C. C., 349; Federer, H., 22, 266; Feferman, S., 412; Feit, W., 150; Feldman, J., 158, 245, 353; Fell, J. M. G., 353; Ficken, F. A., 26; Findlay, G. D., 238; Fleishman, B. A., 393; Flesch, H. K., 238; Floyd, E. E., 11, 398; Ford, L. R., 158, 603; Forsythe, G. E., 8; Fort, M. K., Jr., 171; Fox, C., 383, 397; Frank, E., 40, 565; Frankel, T., 395; Friberg, M. L., 589; Friedberg, R. M., 260; Friedman, B., 359, 589; Fryer, K. D., 594; Fulks, W. B., 565; Fuller, L. E., 350; Fullerton, R. E., 40, 158, 382; Fulton, C. M., 550.

Gál, I. S., 565; Gale, D., 376; Gallie, T. M., Jr., 159; Garrett, J. R., 26; Gates, L. D., Jr., 253; Gehring, F. W., 40; Gelbaum, B. R., 566, 566; Gil de Lamadrid, J., 267, 267, 366, 572; Gillis, J., 167; Gillman, L., 246, 606, 607; Gilmore, P. C., 599; Ginsburg, S., 16; Goffman, C., 151, 354, 566, 566; Goldberg, K., 376; Goldberg, M., 171; Goldberg, R. R., 354; Goldberg, S. I., 257, 258; Goldman, L., 551; Goldman, O., 376; Goldstein, A. A., 567; Goldstine, H. H., 167; Goto, M., 26; Green, L. W., 159; Greenspan, D., 594; Griffin, J. S., Jr., 172; Griffith, H. C., 34; Guenther, P. E., 41; Burk, H. M., 393; Guy, D. L., 41, 159; Guy, W. T., Jr., 6, 41, 167, 589.

Haimo, F., 38, 350, 551; Halberg, C. J. A., Jr., 16; Hale, J. K., 567, 567; Halperin, I., 594; Hamilton, O. H., 398; Hammer, P. C , 45, 359, 361; Hamstrom, M. E., 398; Hanf, W., 402, 551; Harary, F., 149, 597; Harris, B., 2, 2; Harrold, O. G., Jr., 34; Hartmanis, J., 3; Harton, J., Jr., 568; Hausner, A., 383; Hay, G. E., 359; Heerema, N., 26; Heider, L. J., 47, 399, 568; Helfenstein, H. G., 172; Heller, I., 552, 589, 590, 595; Helms, L. L., 603; Henkin, L. A., 412; Henrici, P., 254; Henriksen, M., 38, 48, 607; Hermann, R., 595; Herstein, I. N., 238; Hewitt, E., 376, 377, 384; Higgins, T. P., 6, 167; Hirshman, I. I., Jr., 406, 406; Hocking, J. G., 366; Hodges, J. H., 27, 377, 552; Horne, J. G., Jr., 34, 181; Hsiung, C. C., 258; Huber, A. O., 246; Huckemann, F. C., 384; Huckemann, F., 267; Hughes, D. R., 3, 238, 350, 552; Hughes, H. K., 357; Hummel, J A., 568, 569; Hunt, B., 553; Hunter, R. P., 607, 610; Hutcherson, W. R., 596.

Ikenberry, E., 168; Isbell, J. R., 48, 168, 254, 393.

Jacobson, B., 351; Jaeger, A., 151, 377, 402; James, R. C., 28, 384; Jenner, W. E., 3, 553; Jennings, S. A., 239, 402; Jerison, M., 569; Johnson, E. S., 569; Johnson, G. P., 366; Johnson, G., Jr., 159; Jonsson, B., 403, 553, 554; Jones, B. W., 151; Jurkat, W. B., 42, 407.

Kadison, R. V., 570; Kalisch, G. K., 246; Kalish, D., 261, 261, 261; Kalman, J. A., 403; Karanishi M., 256; Karlin, S., 603; Karush, W., 409, 590; Kato, T., 151; Kazarinoff, N. D., 246, 570; Keedy, M. L., 362; Kelley, J. E., 48; Kelly, L. M., 172; Kemperman, J. H. B., 152, 354; Kendall, D. G., 16, 17, 263, 264; Kerr, C. E., 570; Kiefer, J. C., 177; Kimme, E. G., 42, 413, 603; Kimura, N., 27, 152, 239; Klee, V. L., Jr., 22, 410, 607; Klein, G., 247; Kleinfeld, E., 38; Kobayashi, S., 361, 361, 395, 595; Koch, R. J., 35; Kohls, C. W., 554, 554, 555; Kokoris, L. A., 555; Korevaar, J., 42, 160; Krabbe, G. L., 354; Kramer, V. A , 17, 407; Kreyszig, E., 247, 247, 254, 570, 571, 571, 572, 590; Krickeberg, K., 177, 604; Krule, I. S., 48, 181; Krzywoblocki, M. Z., 46, 355; Kuipers, L., 17; Kulik, S., 160 572; Kuller, R. G., 355; Kunze, R. A., 572; Kuranishi, M., 256; Kyner, W. T., 385. 
Lambek, J., 238; Landin, J., 239, 555; Lane, R. E., 385; Langer, R. E., 248; Lapidus, L., 172; Laugwitz, D., 258; Lax, P. D., 385, 573; Leader, S , 248; Ledermann, W., 152; Leehey, P., 9; deLeeuw, K., 42, 43, 355, 356, 356, 573; Leger, G. F., 378; Lehman, A. B., 378; Lehmer, D. H., 13; Leimanis, E., 6; Leipnik, R. B., 413, 604; LeVeque, W. J., 556; Levin, J. J., 248, 573; Lewis, D. J., 556; Li, T. C. H., 591; Lin, C. C., 394; Littman, W., 255; Livesay, G. R., 366; Lorch, E. R., 173; Lorch, L., 356; Lorentz, G. G., 564, 565; Lotkin, M., 152; Loud, W. S., 31, 248, 249, 385, 574, 574; Ludford, G. S. S., 253; Lukacs, E., 397.

McCandless, B. H., 268; McAuley, L. F., 11; McArthur, C. W., 28; McGregor, J. L., 603; McGregor, J. M., 4; McKelvey, R. W., 6, 570; McLachlan, E. K., 161; McLeod, E. B., 574; MacLane, G. R , 161; MacLane, S., 556, 557; MacNerney, J. S., 29; Malvern, L. E., 591; Mandelbrojt, S., 161, 161; Maness, W. D., 591; Mann, W. R., 6; Mansfield, M. J., 367; Marcus, M. D., 4, 240, 240, 240, 240; Marcus, M., 403, 403; Markus L., 7; Marlow, W. H., 168, 254; Marsaglia, G., 604; Martino, M. A., 249; Marx, I., 255; Masaitis, C., 35; Massey, W. S, 608; Mautner, F. I., 386; Medlin, G. W., 27; Merkes, E. P.,162; Meyer, B. C., 386; Meyers, L. F., 43; Michael, E. A., 23, 414; Miles, E. P., Jr., 31, 31, 162; Miller, D. D., 25; Miller, D. W., 557; Mills, H. D., 9; Mitchell, J., 386; Montague, R., 260, 261, 261, 261, 599; Moore, C. N., 557; Moore, R. A , 7; Morawetz C. S., 394; Morelock J. C., 31, 596; Moser, J., 387; Mostert, P. S., 35, 36, 182, 608; Motzkin, T. S., 14, 14, 259, 558, 574; Moy, S. C., 363; Moyls, B. N., 240, 240; Munkres, J. R., 21, 23.

Nachbar, W., 591; Nash, J. F., 399; Nering, E. D., 351; Neugebauer, C. J., 250; Neumann, B. H., 152; Newman, M., 4, 376; Nicol, C. A., 351; Nijenhuis, A., 361; Nitsche, J., 21, 250, 250; Nitsche, J. C. C., 575, 575, 575, 576; Niven, I., 152; Nohel, J. A., 29, 29; Noll, W., 592; Norton D. A., 550.

Oakley, C. O., 411; Oehmke, R. H., 153; Olum, P., 268, 268; Orey, S., 600; Ornstein, D., 39; Osborn, H. A., 168; Osserman, R., 576; Ostrom, T. G., 173, 596; Owens, O. G., 7; Oxley, T. D., Jr., 357; Oxtoby, J. C., 608.

Pan, T. K., 173, 596; Papakyriakopoulos, C. D., 182, 399, 399, 609; Pardee, O. O., 576; Parzen, E., 264; Payne, L. E., 9, 162, 255; Pearl, M. H., 4; Pearson, B. J., 182; Pease, D. K., 259, 259, 259; Pederson, R. N., 577; Peirce, W. H., 359; Perel, W. M., 27; Perkins, F. W., 577; Perry, N. C., 31, 596; Peters, G. O., 7, 162, 387; Pettis, B. J., 30; Peyerimhoff, A., 42, 163, 387, 407; Phelps, R. R., 596; Phillips, R. S., 577; Pierce, R. S., 14, 14; Pignani, T. J., 163; Poor, V. C., 388, 388; Porcelli, P., 163; Preston, G. C., 404; Price, J. J., 4, 388; Prins, G., 597; Prosser, R. T., 388, 388, 578; Protter, M. H., 578; Pursell, L. E., 609.

Rabin, M. O., 396, 396; Rachford, H. H., 168; Raimi, R. A., 389; Rainich, G. Y., 394; Raleigh, J., 163; Rall, L. B., 407; Ralston, K. E., 558; Rankin, B., 360; Reade, M. O., 156, 164; Rector, R. W., 399; Redheffer, R. M., 18, 408, 408, 408; Ree, R., 239, 402; Regan, F., 584; Reich, E., 578; Reichelderfer, P. V., 578; Reid, W. T., 164, 357; Reiner, I., 5, 27, 239, 555; Reinhart, B. L., 389; Reisel, R. B., 558; Reiter, H. J., 164; Reuter, G. E. H., 17, 263, 264; Reza, F., 592; Riberio, H., 176; Rinehart, R. F., 241; Ritter, I. F., 256; Robinson, D. W., 241; Robinson, G. de B., 378; Robinson, R. M., 600; Rosenberg, A., 379, 558; Rosenblum, M., 30; Roth, J. P., 32, 45, 182, 262, 269; Royden, H. L., 18; Royster, W. C., 32; Rubel, L. A., 251; Rubin, H., 404; Rudin, M. E., 400; Rudin, W., 389, 400, 579, 579; Ryser, H. J., 558.

Sabidussi, G. O., 49, 609, 609; Salzer, H. E., 169, 256; San Soucie, R. L., 404, 559; Saworotnow, P. P., 8, 579; Schaerf, H. M., 165, 579, 580; Schatten, R., 389; Schreiber, M., 251, 251; Schwartz, J. T., 15, 243; Schwartzman, S., 269, 269; Schweitzer, A. R., 262, 262, 262, 362, 363; Scott, W. T., 43; Sedney, R., 9; Segal, I. E., 580; 
Selfridge, J. L., 14, 558; Seligman, G. B., 379; Shapiro, J. M., 178, 364; Shapiro, V. L., 165, 390, 580; Shapley, L. S., 580, 592; Sheffer, I. M., 581; Shields, A. L., 35, 36, 182; Shoenfield, J. R., 390; Silverman, R. J., 547, 547; Simon, A. B., 36; Singer, I. M., 570; Singh, V., 581; Sion, M., 252; Sklar, A., 559; Slye, J. M., 367, 610; Smale, S., 270, 367, 610; Smith, O. K., 18; Smullyan, R. M., 600, 601; Snyder, W. S., 32; Sonneborn, L. M., 23; Spencer, D. E., 10, 395; Spencer, G. L., II, 169; Springer, C. E., 173; Sprinkle, H D, 176, 386; Stamey, W. L., 362; Steger, A., 14; Stein, E. M., 357, 358, 358; Stein, S. K. B., 11; Steinberg, R., 241; Stelson, H. E., 593; Stenberg, W. B., 581; Stewart, B. M., 597; Stone, W. M., 409; Stoneham, R. G., 19; Straus, L. W., 8; Strodt, W., 581, 582, 582, 583; Strohl, G. R., Jr., 11; Strother, W. L., 33, 33; Stroud, A. H., 45; Struik, S. R., 174; Struik, R. R., 379, 379; Sugar, A. C., 19; Supnick, F., 10, 598; Swanson, C. A., 583; Swingle, P. M., 610.

Taam, C. T., 8, 569; Talacko, J. V., 47; Tanimoto, T. T., 597; Tarski, A., 551, 554, 601; Taussky, O., 151; Thompson, G. L., 169; Thompson, G. T., 18; Thorne, R. C., 165, 583; Toralballa, L. V., 44; Trifan, D., 170; Tucker, A. W., 380.

Underwood, R. S., 174, 174; Utz, W. R., Jr., 40, 157, 584.

Valentine, F. A., 598; Varga, R. S., 354; Vaught, R. L., 262, 412, 413, 601; Vazsonyi, A., 409; Vinograde, B., 154; Volkmann, B. W., 153.

Wade, L. I., 606; Wagner, F. J., 393; Wall, D. W., 153, 559; Wall, H. S., 166; Wallace, A. D., 35, 36; Wallen, C. J., 584; Walsh, J. L., 574; Walter, J. H., 404, 560; Wang, H. C., 23; Ward, M., 15, 405; Warncke, D., 598; Warner, S., 390; Wasow, W. R., 409, 584; Wasserman, R. H., 593; Watson, G. L., 151; Weeg, G. P., 154; Weinberger, H. F., 8, 162, 593; Weiss, M. C., 252, 252; Wenjen, C., 28; Whyburn, G. T., 611; Whyburn, W. M., 163; Widom, H., 242, 242; Wilansky, A., 252, 391; Wilcox, C. H., 20, 20, 20, 21; Wilder, R. L., 180; Williams, E., 31, 31, 162; Williams, R. F., 183, 368; Wilson, R. L., 28; Winger, R. M., 598; Wisner, R. J., 411; Wolfe, P. S., 380; Wolfowitz, J., 177; Wong, Y. K., 391, 560, 585, 594; Wright, F. B., Jr., 30; Wright, F. M., 44, 358, 585; Wyman, M., 172.

Yaqub, A. M., 39, 352; Yood, B., 157; 245; 562.

Zelinsky, D., 379; Zemmer, J. L., 242, 349; Zink, R. E., 611; Zondek, B., 253.

\section{RESEARCH PROBLEMS}

Alder, H. L., 76; Bellman, R., 270, 425, 425, 611; Brenner, J. L., 76; Herz, C. S., 76; Klee, V. L., 76, 77; Nash, J., 76; Salzer, H. E., 190, 190; Wallace, A. D., 425, 518, 518 ;

\section{INDEX OF REPORTS OF MEETINGS AND MISCELLANEOUS ARTICLES}

Chern, S. S. See Martin, W. T.

Cohen, L. W. The National Science Foundation Program for Mathematical Sciences, 191.

Klee, V. L., Jr. Reports of Meetings of the American Mathematical Society: November Meeting in Los Angeles, 13; April Meeting in Monterey, 401; Summer Meeting in Seattle, 541.

Martin, W. T., Chern, S. S. and Zariski, O. Scientific Report on the Second Summer Institute. Several Complex Variables, 79.

Meder, A. E., Jr. Report of the Treasurer, 612.

Prager, W. See Youngs, J. W. T.

Roberts, J. H. Reports of Meetings of the American Mathematical Society: November Meeting in Knoxville, 24. 
Schafer, R. D. Reports of Meetings of the American Mathematical Society: October Meeting in College Park, 1; February Meeting in New York, 236; April Meeting in New York, 369.

Youngs, J. W. T. Reports of Meetings of the American Mathematical Society: November Meeting in Milwaukee, 37; Annual Meeting in Houston, 142.

Youngs, J. W. T. and Prager, W. Reports of Meetings of the American Mathematical Society: A pril Meeting in Chicago, 347.

Zariski, O. See Martin, W. T.

\section{INDEX OF BOOK REVIEWS}

Abascal, E. V. Introduction a la geometria diferencial. D. J. Struik, 512.

Alexandrow, A. D. Die innere Geometrie der konvexen Flächen. H. Buseman, 508.

Aleksandrov, P. S. Combinatorial topology. Trans. by H. Komm. Vol. 1. K. Fan, 629.

Bachmann, H. Transfinite Zahlen. F. Bagemihl, 415.

Bagemihl, F. See Bachmann, H.

Ballieu, R. and Simonart, F. Algebre. F. Haimo, 626.

Banach, S. Théorie des opérations linéaires, 277.

Barsotti, I. See Roth. L.

Bartlett, M. S. An introduction to stochastic processes with special reference to methods and applications. D. A. Darling, 73.

Bauer, E. Champs de vecteurs et de tenseurs. Introduction a l'electro-magnetisme. N. Coburn, 512.

Bellman, R. See Coddington, E. A.

Bers, L. An outline of the theory of pseudoanalytic functions, 291.

Bianchi, L. Opere. Vol. 3. Sistemi tripli ortogonali, 74.

Bieberbach, L. Analytische Fortsetzung. R. C. Buck, 184.

Boas, R. P., Jr. Entire functions. J. Korevaar, 57.

- See Doetsch, G., de Losada y Puga, C., Natanson, I. P.

Bögel, K. See Natanson, I. P.

Boothby, W. M. See Hadwiger, H.

Boron, L. F. See Riesz, F., Natanson, I. P.

Bourbaki, N. Espaces vectoriels topologiques. E. Hewitt, 507.

Buck, R. C., See Bieberbach, L.

Busemann, H. See Alexandrow, A. D.

Cartan, H. and Eilenberg, S. Homological algebra. S. MacLane, 615.

Cesari, L. Surface area. T. Rado, 513.

Chevalley, C. The construction and study of certain important algebras. J. Dieudonné, 69.

Chu, L. J. See Stratton, J. A.

Chung, K. L. See Gnedenko, B. V.

Cinquant' anni di relativitd, 1905-1955. Ed. by M. Pantaleo, 76.

Coburn, N. Vector and tensor analysis. D. E. Spencer, 505.

See Bauer, E.

Coddington, E. A. and Levinson, N. Theory of differential equations. R. Bellman, 185.

Collatz, L. Numerische Behandlung von Differentialgleichungen. W. E. Milne, 74.

Computer Directory, June 1955, 75.

Convegno internazionale sulle equazioni lineari alle derivate parziali. Trieste, 25-28 Agosto 1954, 423.

Corbat6, F. J. See Stratton, J. A.

Coxeter, H. S. M. The real projective plans. $2 \mathrm{~d}$ ed., 75. 
Darling, D. A. See Bartlett, M. S., Gnedenko, B. V.

Day, M. M. See Natanson, I. P.

Dieudonné, J. La géométrie des groupes classiques. I. Reiner, 417. See Chevalley, C.

Dini, U. Opere. Vol. 3, 277.

Doetsch, G. Handbuch der Laplace-Transformation. Vol. II: Anwendungen der Laplace-transformation. R. P. Boas, Jr., 628.

Doob, J. L. See Loève, M.

Dresden, A. See van der Waerden, B. L.

Eilenberg, S. See Cartan, H.

Einstein, A. The meaning of relativity. 5th ed., 424.

Fan, K. See Aleksandrov, P. S.

Federer, W. T. Experimental design, theory and application. H. B. Mann, 416.

Feshbach, H. See Morse, P. M.

Furtwängler, P. Allgemeine Theorie der algebraischen Zahlen. Reworked by H. Hasse and W. Jehne. G. Whaples, 73.

Gnedenko, B. V. and Kolmogorov, A. N. Limit distributions for sums of independent and random variables. Translated and annotated by $\mathrm{K}$. L. Chung, D. A. Darling, 50.

Hadwiger, H. Altes und Neues über konvexe Körper, W. M. Boothby, 272.

Haimo, F. See Ballieu, R.

Halmos, P. R. See Hermes, H.

Hasse, H. See Furtwängler, P.

Hay, G. E. See Sokolnikoff, I. S.

Heffter, L. Begrïndung der Funktionentheorie auf alten und neuen Wegen. A. J. Lohwater, 271.

Hermes, H. Einführung in die Verbandstheorie. P. R. Halmos, 189.

Hewitt, E. See Bourbaki, N., Kelley, J. L., Ljusternik, L. A., Natanson, I. P.

Hildebrand, F. B. Introduction to numerical analysis. J. Todd, 624.

Hirsch, K. A. See Kurosh, A. G.

Jehne, W. See Furtwängler, P.

Kac, M. See Morse, P. M.

Kaplan, W. See Lectures on functions of a complex variable.

Kelley, J. L. General topology. E. Hewitt, 65.

Klein, F., Sheppard, W. F., Macmahon, P. A., and Mordell, L. J. Famous problems, 277.

Kolmogorov, A. N. See Gnedenko, B. V.

Komm, H. See Aleksandrov, P. S.

Kopal, Z. Numerical analysis, with emphasis on the application of numerical techniques to problems of infinitesimal calculus in single variables. J. Todd, 624.

Korevaar, J. See Boas, R. P., Jr.

Kurosh, A. G. Theory of groups. Vol. I. Trans. and ed. by K. A. Hirsch, 277.

Lang, S. See Samuel, P.

Lectures on functions of a complex variable. Ed. by W. Kaplan with the assistance of M. O. Reade and G. S. Young, 75.

Levinson, N. See Coddington, E. A.

Little, J. D. C. See Stratton, J. A.

Ljusternik, L. A. and Sobolew, W. I. Elemente der Funktionalanalysis. E. Hewitt, 188.

Loève, M. Probability theory. Foundations. Random sequences. J. L. Doob, 62. 
Lohwater, A. J. See Heffter, L.

de Losada y Puga, C., Curso de análisis matemático. Vol. 3. R. P. Boas, Jr., 74.

MacLane, S. See Cartan, H.

Macmahon, W. F. See Klein, F.

Mann, H. B. See Federer, W. T.

Margenau, H. and Murphy, G. M. The mathematics of physics and chemistry, 424.

Mayer, J. R. Structure of simple fluids, 332.

Miller, G. A. Collected works. Vol. 4, 75.

Milne, W. E. See Collatz, L.

Mordell, L. J. See Klein, F.

Morse, P. M. and Feshbach, H. Methods of theoretical physics, M. Kac, 52. See Stratton, J. A.

Murphy, G. M. See Margenau, H.

Nagy, S. B. See Riesz, F.

Natanson, I. P. Konstruktive Funktionentheorie. Trans. by K. Bögel, R. P. Boas, Jr., 71.

Natanson, I. P. Theory of functions of a real variable. Trans. by L. F. Boron, with the editorial collaboration of and with annotations by E. Hewitt, M. M. Day, 68 .

Nielsen, K. L. Methods in numerical analysis. J. Todd, 624.

Ostmann, H. H. Additive Zahlentheorie, part I: Allgemeine Untersuchungen. H. S. Zuckerman, 515.

Pantaleo, M. See Cinquant' anni di relativitd, 1905-1955.

Rado, T. See Cesari, L.

Reade, M. O. See Lectures on functions of a complex variable.

Reiner, I. See Dieudonné, J.

Riesz, F. and Nagy, S. B., Functional analysis. Trans. by L. F. Boron, 423.

Roth, L. Algebraic threefolds. I. Barsotti, 516.

Samuel, P. Méthodes d'algèbre abstraite en géometrie algébrique. S. Lang, 273.

Schoenfeld, L. See Trost, E.

Second Colloque sur les equations aux derivees partielles. Tenu à Bruxelles du 24 au 26 mai $1954,75$.

Sheppard, W. F. See Klein, F.

Simonart, F. See Ballieu, R.

Sobolew, W. I. See Ljusternik, L. A.

Sokolnikoff, I. S. Mathematical theory of elasticity. 2d ed. G. E. Hay, 416.

Spencer, D. E. See Coburn, N.

Stratton, J. A., Morse, P. M., Chu, L. J., Little, J. D. C., and Corbat6, F. J. Speroidal wave functions, 423.

Struik, D. J. See Abascal, E. V.

Synge, J. L. Relativity: The special theory. A. H. Taub, 420.

Tables of the cumulative binomial probability distribution, 75 .

Taub, A. H. See Synge, J. L.

Thurston, $\mathrm{H}$. A. The number system, 424.

Todd, J. See Kopal, Z., Hildebrand, F. B., Nielson, K. L.

Trost, E., Primzahlen. L. Schoenfeld, 54.

van der Waerden, B. L. Science awakening. Trans. by A. Dresden, 75.

Whaples, G. See Furtwängler, P.

Young, G. S. See Lectures on functions of a complex variable.

Zuckerman, H. S. See Ostmann, H. H. 


\section{INVITED ADDRESSES ${ }^{1}$}

Allendoerfer, C. B., 541.

Barsotti, I., Algebraic group-varieties, 519.

Bers, L., An outline of the theory of pseudoanalytic functions, 291.

Bochner, S.

Buck, R. C., 347.

Chern, S. S., 142.

Chowla, S., 541.

Erdös, P. and Rado, R. A partition calculus in set theory, 427.

Garabedian, P. R., 13.

The mathematical theory of three-dimensional cavities and jets, 219.

$\mathrm{Hu}, \mathrm{S}$. T., 24.

Axiomatic approach to the homotopy groups, 490.

John, F., 369.

Iwasawa, K., 541.

Mayer, J. E., 142.

- Structure of simple fluids, 332.

Olum, P., 1.

Pitcher, E., 236.

Rado, R., See Erdös, P.

Schwartz, J. T., 369.

Rieman's method in the theory of special functions, 531.

Spanier, E. H., 37.

Duality and S-theory, 194.

Wang, H. C., 401.

Whyburn, G. T., 142.

Topological analysis, 204.

${ }^{1}$ Where the title of the address is not given, the reference is to the Report of the Meeting at which the address was given. 